\title{
ALLELOPATHY OF GLIRICIDIA SEPIUM (JAEQ.) KUNTH EX WALP. ON CYAMOPSIS TETRAGONOLOBUS (L.) TAUB. VARIETY SADAFALI.
}

\author{
S.R. Bharate \\ Dadapatil Rajale Arts \& Science College, Adinathnagar. Tal. Pathardi, Dist. Ahmednagar. \\ Corresponding Author: srbharate1969@gmail.com
}

\begin{abstract}
:
The effects of aqueous leaf root stem and Flower extract of different concentrations $(2.5 \%, 5 \%$ and $7.5 \%)$ of plant parts of Gliricidia Sepium (Jaeq.) Kunth Ex Walp from ' Garbhagiri hills of pathardi Tahsil ( longitude 19० 09'N, laditude $75^{\circ} 10^{\prime} \mathrm{E}$ ) were tested on germination and seedling growth of Cyamopsis tetragonolobus (L.) Taub. Variety Sadafali.

Key words: -Extracts, germination, Gliricidia Sepium (Jaeq.) Kunth Ex Walp seedling growth, Cyamopsis tetragonolobus (L.) Taub. Variety Sadafali,.
\end{abstract}

\section{INTRODUCTION:}

Molisch (1937) introduced the word 'Allelopathy' (Greek words: 'allelon' means reciprocal and 'pathos' means that happens to one) for harmful as well as beneficial, biochemical and reciprocal interactions among plants including microorganisms. Allelopathy is defined as "any direct or indirect harmful/useful effect by one plant on another through the synthesis and secretion of chemicals into the environment." Allelopathy is a current area of research. It may be useful in agriculture to increase yield, minimize some problems related to multiple cropping systems, soil productivity and availability of nutrients in soil. Allelopathy, a multidisciplinary subject and research in it will definitely establish a boon in agricultural and forestry production (Narwal and Tauro, 1994 Extracts of Glyricidia maculata $\mathbf{L}$. retard seedling growth parameters of test crop plants viz. cowpea, greengram, black gram, sunflower, horse gram, rice, sorghum, finger millet, mustard, lablab and bean (Patil, 1994).
Volatile oil from the bark of Gliricidia sepium contains methyl-3(E) - pentyl ether, 3methyl 2-butanol, 3-methoxy hexane, 1-(1ethoxyethoxy) - 2-hexane, 2-decanol, Coumarin and hexadecanoic acid (Reddy and Jose, 2010).

\section{MATERIAL \& METHODS:}

Extracts were obtained by crushing plant materials.10\% aqueous extracts (stock solution) obtained from plant parts (leaves, stem, roots, flowers) of Gliricidia sepium(Jacq.)Kunth.ex Steud .Extracts were filtered with muslin cloth and Whatman filter paper No.1, stored in refrigerator and further diluted with distilled water to get extracts of 2.5 $\%, 5 \%$, and $7.5 \%$ (Narwal, 1994). Extracts were further used for bioassay in laboratory conditions.

Effect of these three concentrations on seedling growth parameters viz. seed germination (Ger), Shoot growth (Sg) , Root growth (Rg) and Total seedling growth(TSg) of test crop plant Cyamopsis tetragonolobus L. var. 'Sadafali' were recorded after $5^{\text {th }}$ day. Seeds 
of test plants were surface sterilized with $0.01 \%$ Mercuric chloride followed by thorough washing with distilled water before use.

Ten seeds/ plastic container were germinated in sterilized containers of $12 \mathrm{~cm}$ diameter, using germination paper or Whatman No.1 filter paper. Triplicates of the containers were maintained. $10 \mathrm{ml}$ of extract was added in the Petri dishes/containers containing 10 seeds each. The slight emergence of radical was considered as a sign of germination. Germination percentage was calculated. Photographs were taken with digital camera ('Sony'make). Percentage inhibition or stimulation of 'Ger' (seed Germination), 'Rg' (Root growth), 'Sg' (Shoot growth) and 'TSg' (Total seedling growth) over control was calculated from which graphs were drawn.

\section{RESULT AND DISCUSSION:}

1. Effect of leaf extract of Gliricidia on 'Sadafali' variety: Leaf extracts of Gliricidia exerted significant and concentration correlated inhibition of seedling growth parameters of the 'Sadafali' variety. 'Rg', 'Sg' and 'TSg' were inhibited by 9.31 to $96.36 \%, 51.13$ to $83.56 \%$ and 61.07 to $88.13 \%$ over control respectively (Table NO1, Graph NO1 A).

2. Effect of stem extract of Gliricidia on 'Sadafali' variety: Stem extract of Gliricidia, except slight inhibition of 'Rg' by $8.50 \%$, promoted seedling growth of 'Sadafali' variety. 'Rg', 'Sg' and 'TSg' of the test plant were promoted by 1.34 to $10.07 \%$, 1.28 to $21.15 \%$ and 0.99 to $16.12 \%$ over control respectively. Promotion was not significant and concentration correlated. Seed germination was slightly inhibited by $3.33 \%$ over control (Table NO1, Graph NO 1 B).

3. Effect of root extract of Gliricidia on 'Sadafali' variety: Root extracts of Gliricidia inhibited seedling growth parameters of the test plant significantly at $(\mathrm{P}<0.05 \%)$. 'Ger', 'Rg', 'Sg' and 'TSg' were inhibited by 3.33 to $10.00 \%, 13.81$ to
$68.15 \%, 14.75$ to $33.62 \%$ and 14.38 to $47.05 \%$ over control respectively. Inhibition was concentration correlated (Table NO1, Graph NO $1 \mathrm{C})$.

4. Effect of flower extract of Gliricidia on 'Sadafali' variety: Flower extracts of Gliricidia drastically inhibited seedling growth parameters viz. 'Rg', 'Sg' and 'TSg' by 69.43 to $80.89 \%, 31.79$ to $58.01 \%$ and 47.86 to $67.71 \%$ over control respectively. Seed germination was inhibited slightly by $3.33 \%$.Inhibition was significant $(\mathrm{P}<0.05 \%)$ and concentration correlated (Table NO 1, Graph NO 1 D).

\section{CONCLUSION:}

Leaf, stem, root and flower extracts of Gliricidia sepium (Jacq.) Kunth. ex Steud. inhibited all seedling growth parameters of 'Sadafali' varieties of Cyamopsis tetragonolobus(L) Taub. in an order: Leaf $>$ flowers $>$ root $>$ stem. Leaf extracts exerted more inhibition than flowers, root, and stem extract. Stem extracts except slight inhibition of root growth, promoted seedling growth of 'Sadafali' variety.

\section{REFERENCES:}

Molisch, Hans (1937)"The Influence of one plant on another: Allelopathy'. Edited by Narwal S.S (2001), Translated by L.J.La Fleur \& M.A.Bari Malik. Scientific Publisher, Jodhpur (India).

Narwal, S.S. and Tauro, P.(1994) 'Allelopathy in Agriculture and forestry.' Scientific Publisher, Jodhpur (India).

Patil, B.P. (1994) Effects of Gliricidia maculata L. extracts on field crops. Allelopathy Journal 1(2): 118-120.

Reddy J. and Jose B. (2010) Chemical composition and Antibacterial activity of the volatile oil from the bark of Gliricidia sepium. International Journal of pharmacy and pharmaceutical Sciences. 2(3) 177-179. 
Table No. 1.:- Effect of Leaf, stem, root and Flower extracts of Gliricidia sepium (Jacq.) Kunth ex steud. on seedling growth of varieties of Cyamopsis tetragonolobus (L.) Taub.

\begin{tabular}{|c|c|c|c|c|c|c|c|c|}
\hline \multirow[t]{2}{*}{$\begin{array}{c}\text { Extrac } \\
t\end{array}$} & \multirow{2}{*}{$\begin{array}{c}\text { Cyamops } \\
\text { is } \\
\text { Variety }\end{array}$} & \multirow{2}{*}{$\begin{array}{c}\text { Growth } \\
\text { Paramet } \\
\text { ers }\end{array}$} & \multirow[b]{2}{*}{ Control } & \multicolumn{3}{|c|}{ Extract concentration } & \multirow{2}{*}{$\begin{array}{l}\text { CD at } \\
0.05 \%\end{array}$} & \multirow{2}{*}{$\begin{array}{c}\text { P-value } \\
\text { at } \\
0.05 \%\end{array}$} \\
\hline & & & & $2.50 \%$ & $5 \%$ & $7.50 \%$ & & \\
\hline \multirow{4}{*}{ Leaf } & \multirow{4}{*}{ Sadafali } & $\mathrm{Rg}$ & $\begin{array}{c}2.47 \mathrm{a} \pm \\
0.33\end{array}$ & $\begin{array}{c}0.52 b \pm 0.05 \\
(-9.31)\end{array}$ & $\begin{array}{c}0.21 \mathrm{c} \pm 0.02 \\
(-91.50)\end{array}$ & $\begin{array}{c}0.09 \mathrm{c} \pm 0.01 \\
(-96.36)\end{array}$ & 0.32 & $\begin{array}{c}7.60 \mathrm{E}- \\
19\end{array}$ \\
\hline & & $\mathrm{Sg}$ & $\begin{array}{c}4.44 a \pm \\
0.36\end{array}$ & $\begin{array}{c}2.17 \mathrm{~b} \pm 0.30 \\
(-51.13)\end{array}$ & $\begin{array}{c}1.21 \mathrm{c} \pm 0.12 \\
(-72.75)\end{array}$ & $\begin{array}{c}0.73 \mathrm{~d} \pm 0.10 \\
(-83.56)\end{array}$ & 0.46 & $\begin{array}{c}4.44 \mathrm{E}- \\
19\end{array}$ \\
\hline & & $\mathrm{TSg}$ & $\begin{array}{c}6.91 \mathrm{a} \pm \\
0.65\end{array}$ & $\begin{array}{c}2.69 \mathrm{~b} \pm 0.33 \\
(-61.07)\end{array}$ & $\begin{array}{c}1.42 \mathrm{c} \pm 0.13 \\
(-79.45)\end{array}$ & $\begin{array}{c}0.82 \mathrm{~d} \pm 0.11 \\
(-88.13)\end{array}$ & 0.46 & $\begin{array}{c}8.22 \mathrm{E}- \\
22\end{array}$ \\
\hline & & Ger $\%$ & 93.33 & $\begin{array}{l}93.33 \\
(0.00)\end{array}$ & $\begin{array}{l}93.33 \\
(0.00)\end{array}$ & $\begin{array}{c}70.00 \\
(-25.00)\end{array}$ & & \\
\hline \multirow{4}{*}{ Stem } & \multirow{4}{*}{ Sadafali } & $\mathrm{Rg}$ & $\begin{array}{c}1.49 \mathrm{a} \pm \\
0.19\end{array}$ & $\begin{array}{c}1.64 \mathrm{a} \pm 0.20 \\
(10.07)\end{array}$ & $\begin{array}{c}1.51 \mathrm{a} \pm 0.20 \\
(1.34)\end{array}$ & $\begin{array}{c}1.37 \mathrm{a} \pm 0.18 \\
(-8.05)\end{array}$ & 0.34 & 0.10 \\
\hline & & $\mathrm{Sg}$ & $\begin{array}{c}1.56 \mathrm{a} \pm \\
0.18\end{array}$ & $\begin{array}{c}1.89 \mathrm{ab} \pm 0.17 \\
(21.15)\end{array}$ & $\begin{array}{c}1.58 \mathrm{a} \pm 0.17 \\
(1.28)\end{array}$ & $\begin{array}{c}1.70 \mathrm{a} \pm 0.18 \\
(8.97)\end{array}$ & 0.33 & 0.51 \\
\hline & & $\mathrm{TSg}$ & $\begin{array}{c}3.04 \mathrm{a} \pm \\
0.36\end{array}$ & $\begin{array}{c}3.53 a \pm 0.35 \\
(16.12)\end{array}$ & $\begin{array}{c}3.10 \mathrm{a} \pm 0.36 \\
(1.97)\end{array}$ & $\begin{array}{c}3.07 a \pm 0.36 \\
(0.99)\end{array}$ & 0.67 & 0.73 \\
\hline & & Ger \% & 100.00 & $\begin{array}{c}96.67 \\
(-3.33) \\
\end{array}$ & $\begin{array}{c}100.00 \\
(0.00)\end{array}$ & $\begin{array}{c}101.00 \\
(0.00)\end{array}$ & & \\
\hline \multirow[b]{4}{*}{ Root } & \multirow{4}{*}{ Sadafali } & $\operatorname{Rg}$ & $\begin{array}{l}4.49 \mathrm{a} \pm \\
0.42\end{array}$ & $\begin{array}{c}3.87 \mathrm{~b} \pm 0.40 \\
(-13.81)\end{array}$ & $\begin{array}{c}2.42 \mathrm{c} \pm 0.21 \\
(-46.10)\end{array}$ & $\begin{array}{c}1.43 \mathrm{~d} \pm 0.13 \\
(-68.15)\end{array}$ & 0.59 & $\begin{array}{c}3.96 \mathrm{E}- \\
10\end{array}$ \\
\hline & & $\mathrm{Sg}$ & $\begin{array}{l}7.05 \mathrm{a} \pm \\
0.42\end{array}$ & $\begin{array}{c}6.01 b \pm 0.51 \\
(-14.75)\end{array}$ & $\begin{array}{c}5.41 \mathrm{~b} \pm 0.48 \\
(-23.26)\end{array}$ & $\begin{array}{c}4.68 b \pm 0.39 \\
(-33.62)\end{array}$ & 0.85 & 0.003 \\
\hline & & $\mathrm{TSg}$ & $\begin{array}{c}11.54 \mathrm{a} \pm \\
0.76\end{array}$ & $\begin{array}{c}9.88 \mathrm{~b} \pm 0.83 \\
(-14.38)\end{array}$ & $\begin{array}{c}7.83 c \pm 0.62 \\
(-32.15)\end{array}$ & $\begin{array}{c}6.11 d \pm 0.50 \\
(-47.05)\end{array}$ & 1.29 & $\begin{array}{c}9.07 \mathrm{E}- \\
07\end{array}$ \\
\hline & & Ger \% & 100.00 & $\begin{array}{c}96.67 \\
(-3.33)\end{array}$ & $\begin{array}{c}96.67 \\
(-3.33)\end{array}$ & $\begin{array}{c}90.00 \\
(-10.00)\end{array}$ & & \\
\hline \multirow{4}{*}{ Flowe } & \multirow{4}{*}{ Sadafali } & $\mathrm{Rg}$ & $\begin{array}{c}6.28 \mathrm{a} \pm \\
0.79\end{array}$ & $\begin{array}{c}1.92 \mathrm{~b} \pm 0.30 \\
(-69.43)\end{array}$ & $\begin{array}{c}1.35 \mathrm{~b} \pm 0.19 \\
(-78.50)\end{array}$ & $\begin{array}{c}1.20 \mathrm{~b} \pm 0.33 \\
(-80.89)\end{array}$ & 0.86 & $\begin{array}{c}1.90 \mathrm{E}- \\
13\end{array}$ \\
\hline & & $\mathrm{Sg}$ & $\begin{array}{c}8.43 \mathrm{a} \pm \\
0.60\end{array}$ & $\begin{array}{c}5.75 b \pm 0.57 \\
(-31.79)\end{array}$ & $\begin{array}{c}4.98 \mathrm{~b} \pm 0.61 \\
(-40.93)\end{array}$ & $\begin{array}{c}3.54 \mathrm{c} \pm 0.48 \\
(-58.01)\end{array}$ & 1.06 & $\begin{array}{c}2.25 \mathrm{E}- \\
07\end{array}$ \\
\hline & & TSg & $\begin{array}{c}14.71 \mathrm{a} \pm \\
1.21\end{array}$ & $\begin{array}{c}7.67 b \pm 0.79 \\
(-47.86)\end{array}$ & $\begin{array}{c}6.33 b \pm 0.78 \\
(-56.97)\end{array}$ & $\begin{array}{c}4.75 b c \pm 0.71 \\
(-67.71)\end{array}$ & 1.68 & $\begin{array}{c}3.45 \mathrm{E}- \\
12\end{array}$ \\
\hline & & Ger \% & 100.00 & $\begin{array}{c}100.00 \\
(0.00)\end{array}$ & $\begin{array}{c}86.67 \\
(-13.13)\end{array}$ & $\begin{array}{c}86.67 \\
(-13.13)\end{array}$ & & \\
\hline
\end{tabular}

Data presented are means of three replicates; values within the same row with different letters are significantly different at $0.05 \%$ P-level by Single factor ANOVA test followed by CD \& Tukey's test. [Figures in parentheses indicate \% stimulation (+) and \% inhibition (-) over control; Sg: shoot growth, Rg: root growth, TSg: total seedling growth and Ger: seed germination:] 
Graph No: 1 Effect of Leaf, stem, root and Flower extracts of Gliricidia sepium (Jacq.) Kunth ex steud. on seedling growth of varieties of Cyamopsis tetragonolobus (L.) Taub.
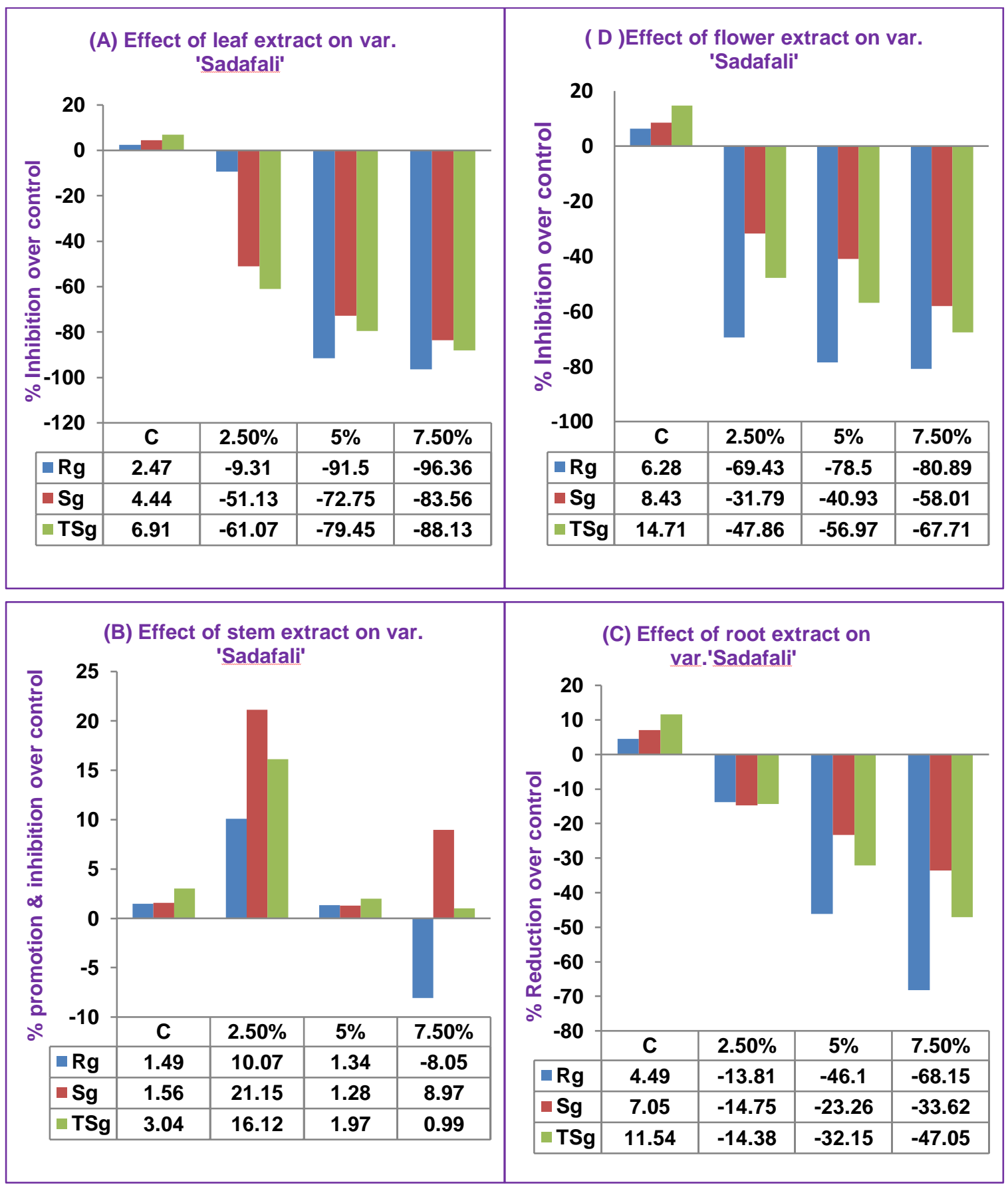

[Where Rg: root growth, Sg: shoot growth, TSg: total seedling growth, figures indicate \% inhibition $(-)$ and $\%$ promotion (+) over control, (C), 2.50 to $7.50 \%$ : Extract concentration] 